\title{
OPEN Identification and characterization of CYPs induced in the Drosophila antenna by exposure to a plant odorant
}

\author{
Shane R. Baldwin ${ }^{1,3,7}$, Pratyajit Mohapatra ${ }^{1,7}$, Monica Nagalla ${ }^{1,5}$, Rhea Sindvani ${ }^{1,6}$, \\ Desiree Amaya ${ }^{1,4}$, Hope A. Dickson ${ }^{1} \&$ Karen Menuz ${ }^{1,2 \bowtie}$
}

Members of the cytochrome p450 (CYP) enzyme family are abundantly expressed in insect olfactory tissues, where they are thought to act as Odorant Degrading Enzymes (ODEs). However, their contribution to olfactory signaling in vivo is poorly understood. This is due in part to the challenge of identifying which of the dozens of antennal-expressed CYPs might inactivate a given odorant. Here, we tested a high-throughput deorphanization strategy in Drosophila to identify CYPs that are transcriptionally induced by exposure to odorants. We discovered three CYPs selectively upregulated by geranyl acetate using transcriptional profiling. Although these CYPs are broadly expressed in the antenna in non-neuronal cells, electrophysiological recordings from CYP mutants did not reveal any changes in olfactory neuron responses to this odorant. Neurons were desensitized by pre-exposing flies to the odorant, but this effect was similar in CYP mutants. Together, our data suggest that the induction of a CYP gene by an odorant does not necessarily indicate a role for that CYP in neuronal responses to that odorant. We go on to show that some CYPs have highly restricted expression patterns in the antenna, and suggest that such CYPs may be useful candidates for further studies on olfactory CYP function.

Odors fluctuate rapidly in the environment, and olfactory receptor neurons are able to respond to this dynamic information. Odorants dissolve into the fluid surrounding olfactory receptor neurons, where they bind and activate receptors on the neuron dendrites. It has long been observed that olfactory tissues abundantly express a large variety of xenobiotic metabolizing enzymes, such as esterases, cytochrome p450s (CYPs), aldehyde oxidases, and glutathione-S-transferases (GSTs) ${ }^{1-3}$. More than 30 years ago, it was first postulated that these enzymes may contribute to olfactory signaling by terminating the olfactory signal through the conversion of odorants into inactive metabolites ${ }^{3}$. Since then, studies in humans, rodents, and insects have shown that broad pharmacological blockers of such Odorant Degrading Enzymes (ODEs) can affect the responses of olfactory receptor neurons to odorants ${ }^{4-6}$. However, there are still relatively few examples of specific ODE genes that are known to affect neuronal responses in vivo. Our best understanding of the olfactory function of ODEs is in insects, where several ODEs that metabolize pheromones have been identified and studied in vitro.

CYPs are found in nearly all organisms and constitute one of the largest gene families ${ }^{7}$. Members of this large and rapidly evolving enzyme family carry out diverse functions on endogenous and foreign substrates, including hormone synthesis and xenobiotic detoxification. In Drosophila, there are 87 CYPs genes, which are expressed in a variety of tissues ${ }^{8-10}$. More than 50 CYPs are expressed in the antenna, their primary olfactory tissue ${ }^{11,12}$. As in other insect species, expression of some Drosophila CYPs is highly enriched in the antenna compared to other tissues, suggesting an olfactory function ${ }^{12-14}$. However, no Drosophila CYPs have been reported to contribute to olfactory signaling. In other insects, biochemical studies indicate a role for specific CYPs in degrading particular odorants and pheromones in vitro ${ }^{15,16}$, but to our knowledge, there is only one example of a specific CYP gene directly shown to affect olfactory neuron responses to a particular odorant in vivo ${ }^{17}$.

\footnotetext{
${ }^{1}$ Department of Physiology and Neurobiology, University of Connecticut, Storrs, CT 06269, USA. ${ }^{2}$ Institute for Systems Genomics, University of Connecticut, Storrs, CT 06269, USA. ${ }^{3}$ Present address: MBF Bioscience, Williston, VT 05495, USA. "Present address: Biomedical Sciences Program, Thomas Jefferson University, Philadelphia, PA 19107, USA. 5 Present address: Feinberg School of Medicine, Northwestern University, Chicago, IL 60611, USA. ${ }^{6}$ Present address: School of Medicine, University of Connecticut, Farmington, CT 06032, USA. ${ }^{7}$ These authors contributed equally: Shane R. Baldwin and Pratyajit Mohapatra. ${ }^{\circledR}$ email: karen.menuz@uconn.edu
} 
A critical hurdle in the identification of CYPs with olfactory roles is that the substrates of most CYPs in Drosophila, the most genetically amenable organism, are unknown ${ }^{9,18}$. Further, many insect CYPs with roles in xenobiotic metabolism act on multiple substrates ${ }^{19}$, and thus the identification of one substrate does not preclude another. Additionally, it is not yet possible to predict substrates from CYP sequences alone because they have highly divergent protein sequences, despite their conserved three dimensional structure ${ }^{20}$. It is estimated that one-third of the Drosophila CYP family is involved in metabolizing environmental xenobiotics based on their evolutionary instability, enrichment in tissues commonly associated with detoxification, and ability to be induced by xenobiotics ${ }^{18,21}$. Of these, $\sim 90 \%$ are expressed in the Drosophila antenna ${ }^{11,12}$. Given this large number of candidates and the hundreds of odors known to activate Drosophila olfactory neurons, it is difficult to predict which CYP may act on which odorant.

In Drosophila as in other insects, the presence of xenobiotics leads to the transcriptional upregulation of selective CYPs, a process referred to as induction ${ }^{22-26}$. Although CYP induction is generally measured following xenobiotic feeding or physical contact, some recent studies in beetles, locusts, and moths have indicated that exposure to volatile odorants can induce CYP expression in the antenna ${ }^{27-30}$. In this study, we identified three CYPs induced by exposure to the odorant geranyl acetate in Drosophila, and examined whether the induced CYPs might contribute the detection of that odorant by olfactory neurons.

\section{Results}

Odorant exposure leads to transcriptional upregulation of a small subset of CYPs. A recent study by von der Weid, et al. employed transcriptional deorphanization to identify mammalian odorant receptors that respond to particular odorants in an unbiased fashion ${ }^{31}$. The same study also demonstrated that activated olfactory receptors are transcriptionally downregulated in Drosophila following exposure to the odorants geranyl acetate and ethyl lactate (activating Or82a and Or67c receptors, respectively). We wondered whether this approach could be used to identify CYPs that utilize particular odorants as substrates. To begin, we mimicked the described odorant exposure paradigm by housing flies in vials with a high concentration (5\%) of geranyl acetate, ethyl lactate, or the solvent DMSO for five hours. Although the stimulation paradigm is nonphysiological, it was used with the goal of strongly driving transcriptional changes. We then harvested three biological replicates of antennal RNA from each condition and used the RNA for RNASeq transcriptional profiling (Fig. 1a). Using the EdgeR program for differential gene expression analysis, we analyzed olfactory receptor expression following odorant exposure and compared this to levels following DMSO exposure (Supplementary Data S1). Or82a expression trended towards lower levels following geranyl acetate exposure ( $75 \%$ of controls), and Or67c expression trended towards lower levels following ethyl lactate exposure ( $\sim 83 \%$ of controls). These changes were not statistically significant, but they are similar to the magnitudes of transcriptional changes reported by the von der Weid et al. study (Or82a 60\% of controls; Or $67 c \sim 70 \%$ of controls), which employed larger sample sizes to detect differences ${ }^{31}$. Overall, these data suggest that our odorant exposure paradigm is effective for driving transcriptional changes.

Interestingly, our dataset also revealed that three CYPs, Cyp6a8, Cyp6 $d 5$, and Cyp4p1, were induced by geranyl acetate exposure compared to either DMSO or ethyl lactate exposure with FDR $<0.15$ (Fig. $1 \mathrm{~b}$ and Supplementary Data S1). The expression of other CYPs was unaffected by geranyl acetate exposure, and no CYPs were induced by ethyl lactate. Cyp $6 a 8$ showed the highest fold-change $(\sim 15$-fold) following exposure to geranyl acetate, and it had the lowest DMSO expression (37 RPM) of the geranyl-acetate sensitive CYPs. Cyp4p1 had the smallest fold-change ( 2.7-fold), and the highest baseline expression (486 RPM), whereas Cyp6d5 was intermediate in both measures. Although geranyl acetate is not a preferential CYP substrate, there is at least report of a CYP that binds and acts on this compound ${ }^{32}$. We therefore decided to study the CYPs that were transcriptionally regulated by this plant odorant.

We first measured the timecourse of antennal CYP induction following geranyl acetate exposure using qRTPCR. We found that transcript levels of $C y p 4 p 1$ and $C y p 6 d 5$ rose sharply during the first two hours of geranyl acetate exposure, and then fell gradually over the next $10 \mathrm{~h}$ (Fig. 1c). This is in agreement with a timecourse previously observed for CYP induction in Drosophila ${ }^{24}$. Given the peak response at two hours, further experiments were carried out at this time point. Accurate detection of Cyp6a8 expression was difficult due its relatively high $\mathrm{C}_{\mathrm{T}}$ value reflecting its low baseline expression, and it was not analyzed for these experiments.

We next examined the dose-response relationship for CYP induction by geranyl acetate exposure. We found that $>0.1 \%$ geranyl acetate was needed to elicit changes in Cyp $6 d 5$ or Cyp4p1 expression in the antenna, and that their upregulation did not saturate even at $10 \%$ geranyl acetate (Fig. 1d).

Finally, we validated the selective induction of Cyp4p1, Cyp6d5, and Cyp6a8 by geranyl acetate by exposing flies to $5 \%$ geranyl acetate, ethyl lactate, or DMSO for two hours and quantifying antennal gene expression with qRT-PCR. Consistent with our RNASeq data (Fig. 1b), Cyp4p1, Cyp6d5, and Cyp6a8 were upregulated by geranyl acetate exposure, but not by exposure to ethyl lactate (Fig. 1e). In contrast, expression levels of three control CYPs, Cyp6a17, Cyp28a5 and Cyp9b1, were unchanged following either geranyl acetate or ethyl lactate exposure. Thus, geranyl acetate induces a selective upregulation of $C y p 4 p 1, C y p 6 d 5$, and Cyp6a8, which we will refer to as GA-induced Cyps.

Cyp4p1 and Cyp6d5 are broadly expressed in non-neuronal cells. We investigated the localization of GA-induced CYPs in antennal sections using fluorescence in situ hybridization (FISH). Anti-sense FISH probes detected specific expression of Cyp4p1 and Cyp6d5, but not Cyp6a8, the CYP with lowest antennal expression (Fig. 1b and Supplementary Fig. S1). Staining antennal sections from flies exposed to geranyl acetate revealed that both $C y p 4 p 1$ and $C y p 6 d 5$ are broadly expressed throughout the antenna (Fig. 2a,b). Given that neu- 
a

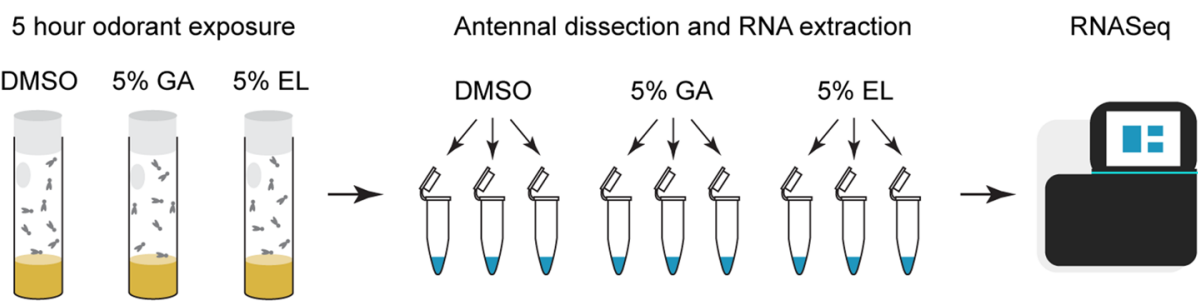

b

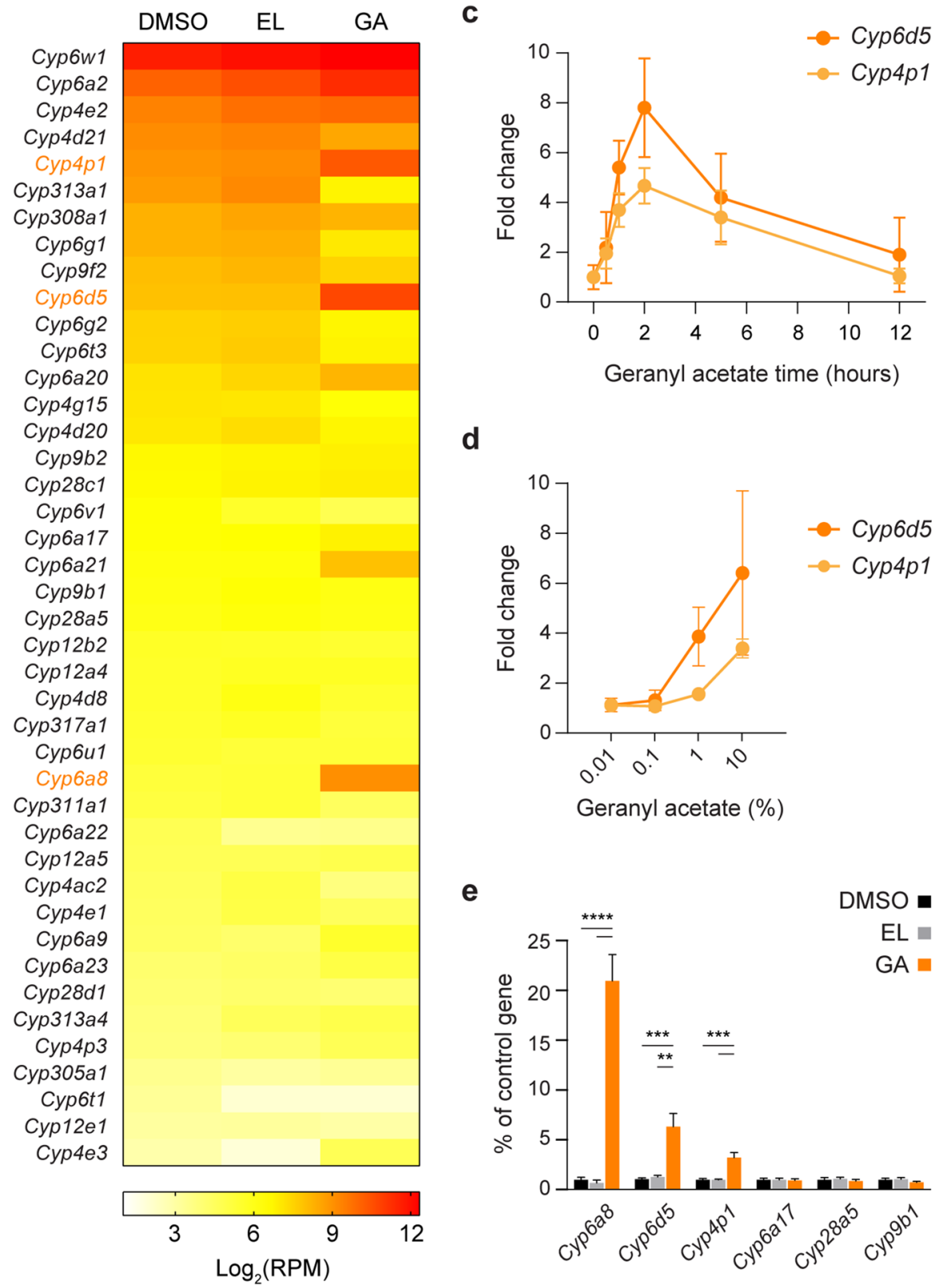

Figure 1. Three CYPs are consistently and specifically upregulated by geranyl acetate exposure. (a) Schematic of RNASeq workflow. Flies were exposed for $5 \mathrm{~h}$ to either DMSO, 5\% geranyl acetate (GA), or $5 \%$ ethyl lactate (EL). Three biological replicates of $\sim 200$ antenna were collected and used for RNASeq analysis. (b) A heat map showing the average expression in $\log _{2}(\mathrm{RPM})$ of each of the 42 antennal-expressed CYP genes in each odorant exposure condition $(n=3)$. GA-induced CYPs are in orange font. (c) The antennal expression of Cyp6d5 and Cyp4p1 detected with qRT-PCR following exposure to 5\% geranyl acetate for varying lengths of time. Expression is normalized to expression at $0 \mathrm{~h}$ of exposure for each gene $(\mathrm{n}=4)$. $(\mathrm{d})$ The antennal expression of Cyp $6 d 5$ and Cyp4p1 detected with qRT-PCR following $2 \mathrm{~h}$ of exposure to varying concentrations of geranyl acetate in DMSO. Expression is normalized to DMSO exposure alone $(n=5-6)$. (e) The expression of six CYPs following 2-h exposure to $5 \%$ geranyl acetate and measured with qRT-PCR $(n=5)$. Data were analyzed by one-way ANOVAs followed by Tukey post-hoc tests. Statistical significance is presented as ${ }^{* *} p<0.01,{ }^{* *} p<0.001$, and ${ }^{* * * *} p<0.0001$. Other comparisons were not significant $(p>0.05)$. For $(\mathbf{c}-\mathbf{e})$, data are shown as the mean \pm SEM. 
a

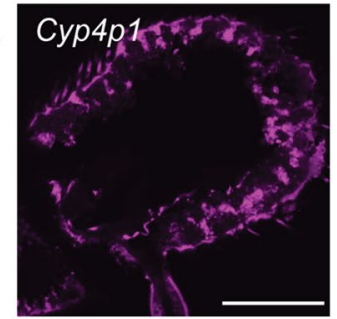

b

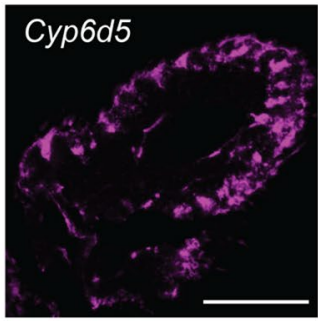

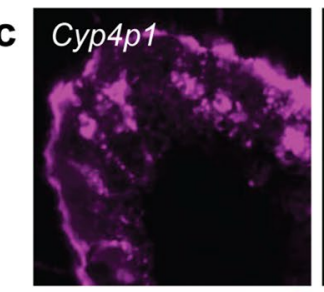
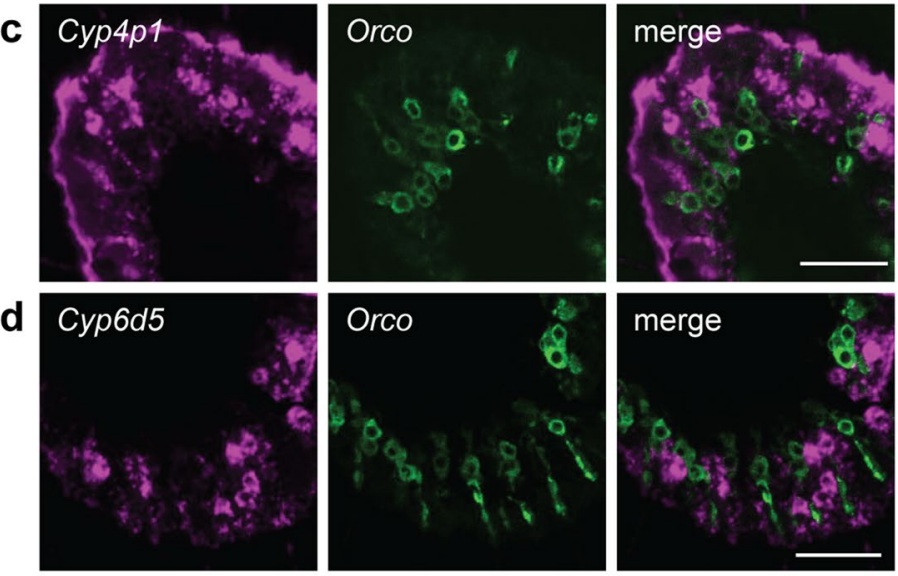

e
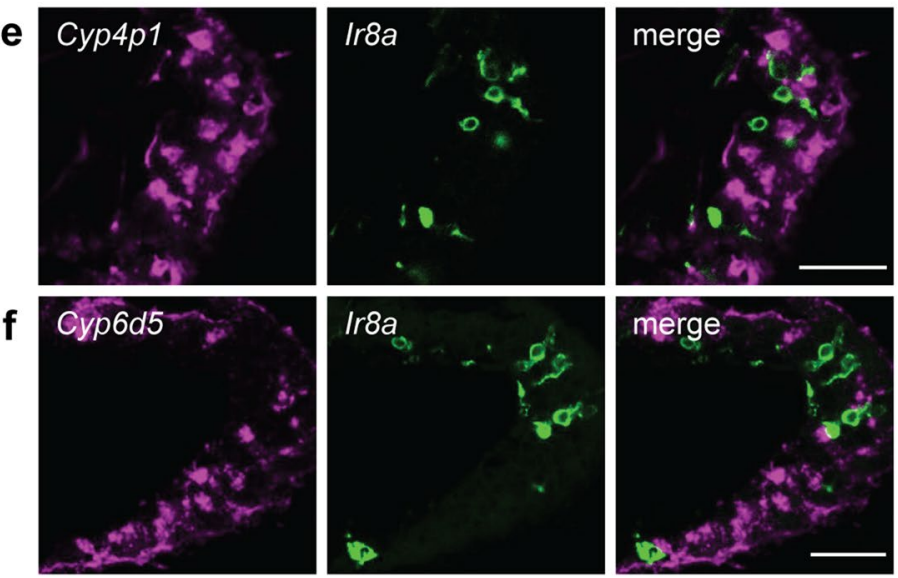

Figure 2. Cyp4p1 and Cyp6d5 are broadly expressed and are not found in olfactory neurons. (a,b) Antennal sections from flies exposed to geranyl acetate for two hours and labeled with fluorescence in situ hybridization (FISH) probes (magenta) for (a) Cyp4p1 or (b) Cyp6d5. Scale bars, $50 \mu \mathrm{m}$. (c,d) Antennal sections from geranylacetate exposed Orco $>$ GFP flies labeled with an anti-GFP antibody (green) and FISH probes (magenta) for (c) Cyp4p1 or (d) Cyp6d5. Scale bars, $20 \mu \mathrm{m}$. (e,f) Similar to (c,d), but with Ir8a> GFP flies.

rons responding to geranyl acetate (Or82 $\mathrm{a}^{+}$neurons in ab5 sensilla) are only found in one of $\sim 20$ classes of olfactory sensilla ${ }^{33-36}$, this suggests that these CYPs are not exclusively expressed in geranyl acetate sensitive sensilla.

Within the antenna, auxiliary cells are thought to express Odorant Degrading Enzymes (ODEs), including CYPs, but few studies have examined their cellular localization. In mammals, ODEs have been detected in both olfactory neurons and surrounding support cells ${ }^{3}$. Might some CYPs likewise be expressed in olfactory neurons in addition to their expected expression in auxiliary cells? To address this question, we examined Cyp4p1 and Cyp6d5 expression with FISH on antennal sections from flies in which subsets of olfactory neurons were labeled with GFP driven by transgenic reporters. In Drosophila, $>70 \%$ of olfactory neurons express Orco, a co-receptor required for the function of OR type olfactory receptors ${ }^{37,38}$. We found that $\mathrm{Orco}^{+}$neurons do not co-express either Cyp4p1 or Cyp6d5 (Fig. 2c,d). An IR-family co-receptor, Ir8a, is expressed by nearly all olfactory neurons that do not express $\mathrm{Orco}^{37}$. Like Orco ${ }^{+}$neurons, Ir8a ${ }^{+}$neurons express neither Cyp4p1 nor Cyp6d5 (Fig. 2e,f). Taken together, our data indicate that the GA-induced CYPs are not expressed in olfactory neurons.

Loss of GA-induced CYPs does not affect electrophysiological responses of olfactory neurons. Do Cyp4p1, Cyp6a8, and Cyp6d5 contribute to olfactory responses mediated by geranyl acetate sensitive 
a

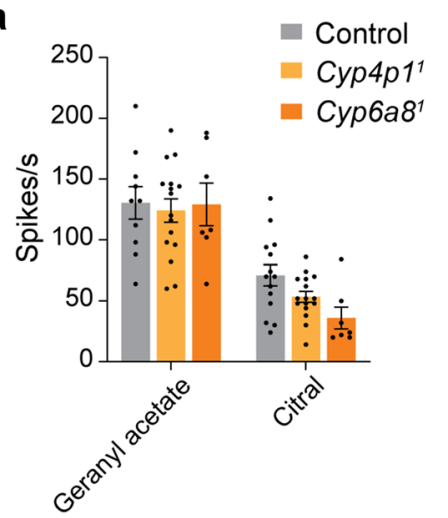

b

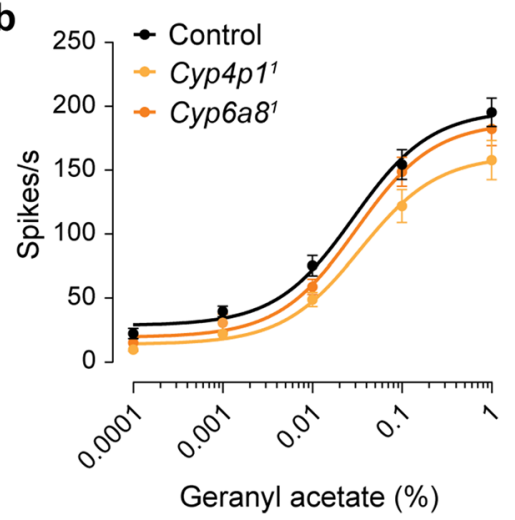

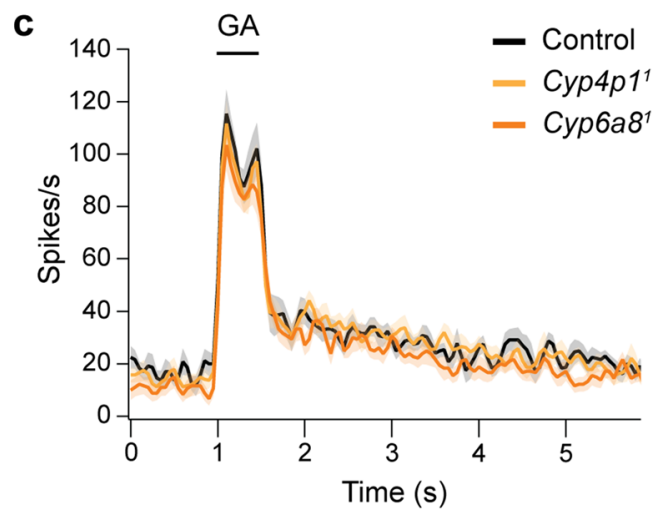

Figure 3. Odorant responses of GA-sensitive neurons are unaffected in $C y p 4 p 1^{1}$ and $C y p 6 a 8^{1}$ mutants. (a) Responses to odors that activate the ab5A neuron, geranyl acetate $(0.1 \%)$ and citral $(0.1 \%)$, are similar in Cyp $4 p 1^{1}$ and Cyp6a8 $8^{1}$ mutants as in controls. Bar graphs depict the mean \pm SEM overlaid with the individual data points. Data analyzed with two-way ANOVA followed by Tukey's post-hoc tests $(\mathrm{n}=8-17$ sensilla each, $p>0.05$ for all comparisons). (b) Dose-response curve of spiking responses to geranyl acetate. Dose response curves show the mean \pm SEM and the curve fit to the Hill equation. The $\mathrm{EC}_{50} \mathrm{~s}$ were not significantly different between the three genotypes (control 0.029\% $\pm 0.008, C y p 4 p 1^{1} 0.034 \% \pm 0.013 \%, C y p 6 a 8^{1} 0.031 \% \pm 0.010 \%$, one-way ANOVA, $\mathrm{n}=8-15$ sensilla each). (c) Peristimulus time histograms (PSTHs, mean \pm SEM) show spiking responses to a $500 \mathrm{~ms}$ pulse of geranyl acetate $(0.01 \%)$. Each trace (colored line) represents the mean of 8-15 recordings taken from the data for the dose-response curve in (b).

olfactory neurons? We hypothesized that if these CYPs inactivate geranyl acetate, their absence might prolong or amplify neuronal spiking responses to this odor. To test this possibility, we generated mutants for each of the three GA-induced CYPs using CRISPR/Cas9 engineering to replace much of the coding region of each gene with a 3xP3-DsRed reporter (Methods). The conserved CYP consensus sequence containing a required cysteine that interacts with the heme iron was deleted in each mutant, presumably rendering each CYP non-functional ${ }^{9,39}$. Following PCR validation, the deletions were outcrossed for at least 10 generations to a standardized genetic background. The $C y p 4 p 1^{1}$ and $C y p 6 a 8^{1}$ mutants were viable, but we were unable to obtain $C y p 6 d 5^{1}$ homozygous mutants.

We used single-sensillum recordings (SSR) to examine the spiking responses of geranyl acetate-sensitive olfactory neurons in ab5 sensilla in the Cyp4p $1^{1}$ and $C y p 6 a 8^{1}$ flies. In Drosophila, only Or82 $\mathrm{a}^{+}$neurons in ab5 sensilla respond strongly to geranyl acetate ${ }^{33-36}$. We found that responses to brief $(500 \mathrm{~ms})$ pulses of geranyl acetate were similar in the two CYP mutants as in the genetic background control (Fig. 3a). Likewise, responses to another odorant detected by Or82a, citral, were unaffected by the loss of either Cyp4p1 or Cyp6a8 (Fig. 3a). We next investigated spiking responses to geranyl acetate using a dose-response curve, and found that the response $\mathrm{EC}_{50}$ in control flies was not statistically different from either CYP mutant (Fig. 3b).

Data in Fig. 3a,b quantified the cumulative spiking response over the $500 \mathrm{~ms}$ response period. However, this could obscure more subtle changes in the response kinetics. We therefore inspected the instantaneous firing frequency over a longer time period using peristimulus time histograms. The kinetics of the geranyl acetate responses were indistinguishable from controls in the Cyp4p $1^{1}$ and Cyp6a $8^{1}$ mutants (Fig. 3c).

We next examined responses to repetitive stimuli, for which odorant inactivation may be more critical compared to responses to single pulses. In the absence of odorant removal, modeling suggests that odorants accumulate in the sensillum lymph and would not reflect environmental levels ${ }^{40}$. We therefore assessed spiking responses to paired-pulses and trains of geranyl-acetate stimuli in Cyp4p $1^{1}$ mutants. First, we applied two $500 \mathrm{~ms}$ pulses of geranyl acetate at 10,3, or $1.5 \mathrm{~s}$ intervals, and we measured the paired-pulse ratio for each sensillum as the response to the second pulse divided by the first pulse. At each inter-stimulus interval (ISI), the paired-pulse ratio was $\sim 1$ in both control and $C y p 4 p 1^{1}$ flies, and their ratios were not statistically different from each other (Fig. $4 \mathrm{a}-\mathrm{d}$ ).

We also applied a train of five $200 \mathrm{~ms}$ geranyl acetate pulses at $1 \mathrm{~Hz}$, and measured the response to each pulse relative to the response to the first pulse for that sensillum. Although responses trended slightly lower with increasing pulse number, no changes were found in the response of $C y p 4 p 1^{1}$ mutants relative to controls (Fig. 4e,f).

Reduced spiking responses to geranyl acetate following odorant exposure are independent of GA-induced CYPs. Although our data do not reveal a role for GA-induced CYPs under baseline conditions, we wondered whether they might play a greater role following their upregulation by geranyl acetate exposure. For example, the tonic presence of geranyl acetate might desensitize Or82a receptors, reducing its odorant sensitivity, and the absence of odorant clearance might exacerbate this effect. To test this possibility, we pre-exposed flies to $5 \%$ geranyl acetate for two hours and examined ab5 spiking responses using electrophysiological recordings within $2 \mathrm{~h}$ following the odorant exposure. Strikingly, responses to both $0.01 \%$ and $0.1 \%$ geranyl acetate in control flies were reduced $\sim 70 \%$ by the prolonged exposure to geranyl acetate (Fig. 5a,b). 
a 10s ISI

control control

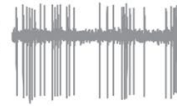

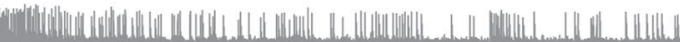

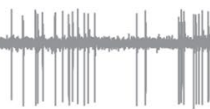

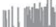

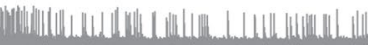

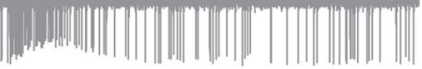

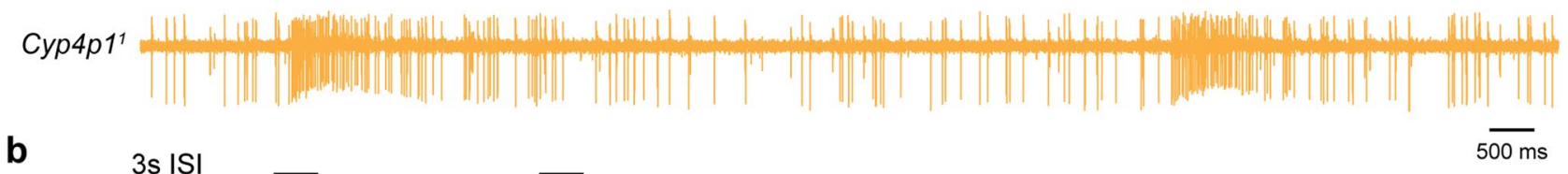

b 3s ISI

control<smiles>C#CC#C</smiles>

Cур $4 p 1^{1}$<smiles>C#CC12C3C4C5C3C1C5C42</smiles>

C

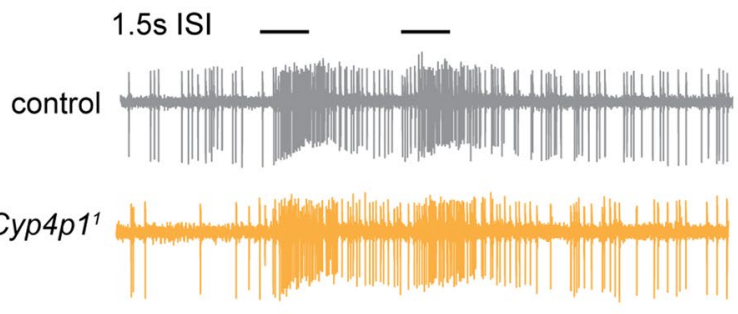

$\overline{500 \mathrm{~ms}}$

e

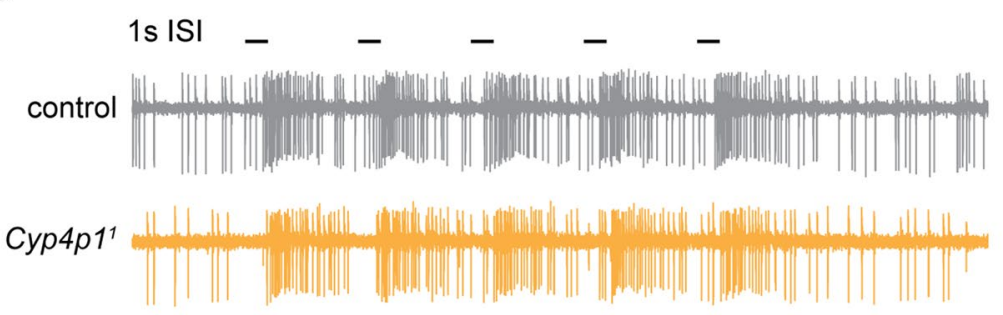

$20 \overline{\mathrm{ms}}$ d

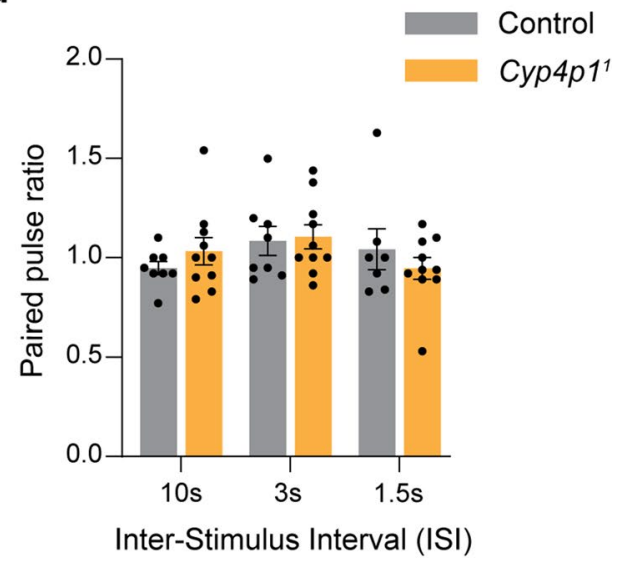

f

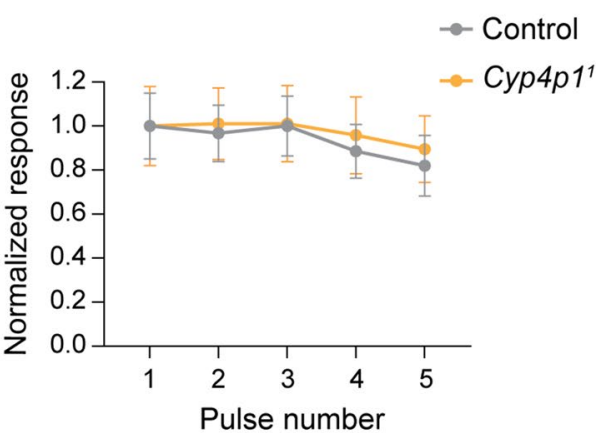

Figure 4. Responses to repetitive geranyl acetate pulses are unaffected in $C y p 4 p 1^{1}$ mutants. (a) SSR traces from control (gray) and Cyp4p $1^{1}$ (orange) flies showing responses to paired $500 \mathrm{~ms}$ pulses of geranyl acetate $(0.01 \%)$ given $10 \mathrm{~s}$ apart. The time at which pulses were given is shown with a black bar. (b,c) Similar to (a), but with inter-stimulus intervals (ISI) of (b) $3 \mathrm{~s}$ or (c) $1.5 \mathrm{~s}$. (d) Quantification of the paired-pulse ratio at each ISI. Bar graphs depict the mean \pm SEM overlaid with the individual data points. The ratios were similar in control and Cyp4p1 $1^{1}$ flies (two-way ANOVA, $p>0.05, \mathrm{n}=7-10$ each). (e) SSR traces from control (gray) and Cyp4p1 $1^{1}$ (orange) flies showing responses to a train of five $200 \mathrm{~ms}$ pulses of geranyl acetate $(0.01 \%)$ given $1 \mathrm{~s}$ apart. (f) Spiking responses to the geranyl acetate train as a function of the pulse number. Responses for each sensillum were normalized to the mean response to the first pulse for that genotype. The graph shows the mean $\pm \mathrm{SEM}$. Responses slightly decrease with increasing pulse number. The responses were similar in control and Cyp4p $1^{1}$ flies (two-way repeated measures ANOVA, $p>0.05, \mathrm{n}=5$ and 7 , respectively).

We also examined responses in $C y p 4 p 1^{1}$ and $C y p 6 a 8^{1}$ flies pre-exposed to geranyl acetate. They exhibited suppressed spiking responses to geranyl acetate, similar to controls (Fig. 5b-e). Therefore, the mechanism by which odorant exposure leads to reduced responses is independent of the expression of these two CYPs.

An alternative strategy to identify CYPs with roles in Drosophila olfaction? Together, our data indicate that although the expression of some CYPs is induced by geranyl acetate, they are unlikely to play a role in olfactory signaling mediated by this odorant. This suggests that transcriptional deorphanization may not be a useful approach to identify CYPs with roles in Drosophila olfaction because transcriptional upregulation is not tightly coupled to function. We wondered whether investigating the expression pattern of antennal CYPs may be a useful strategy to explore this question in the future. Although the GA-induced CYPs we identified are 
a

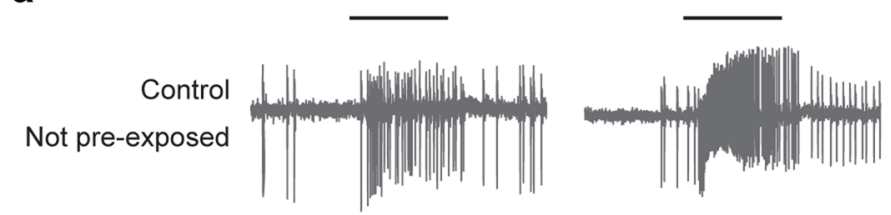

b

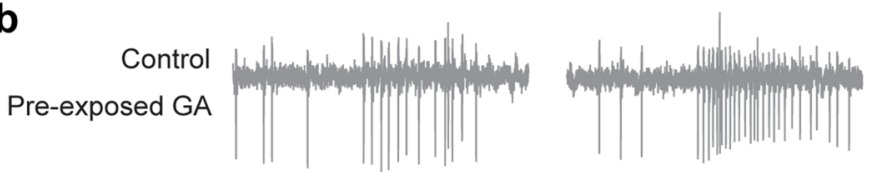

C
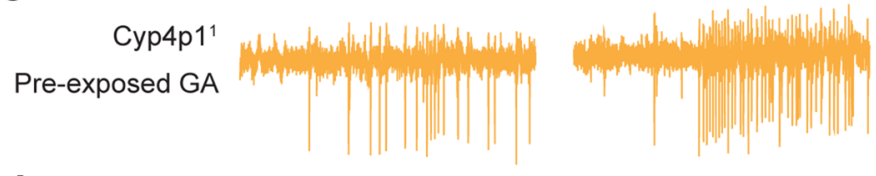

d
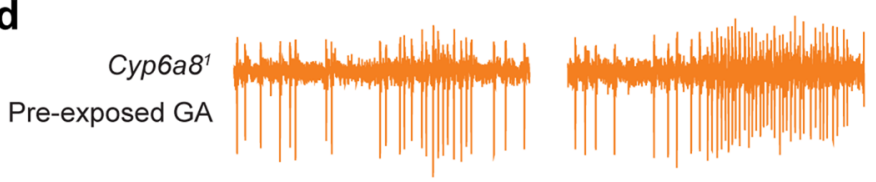

e

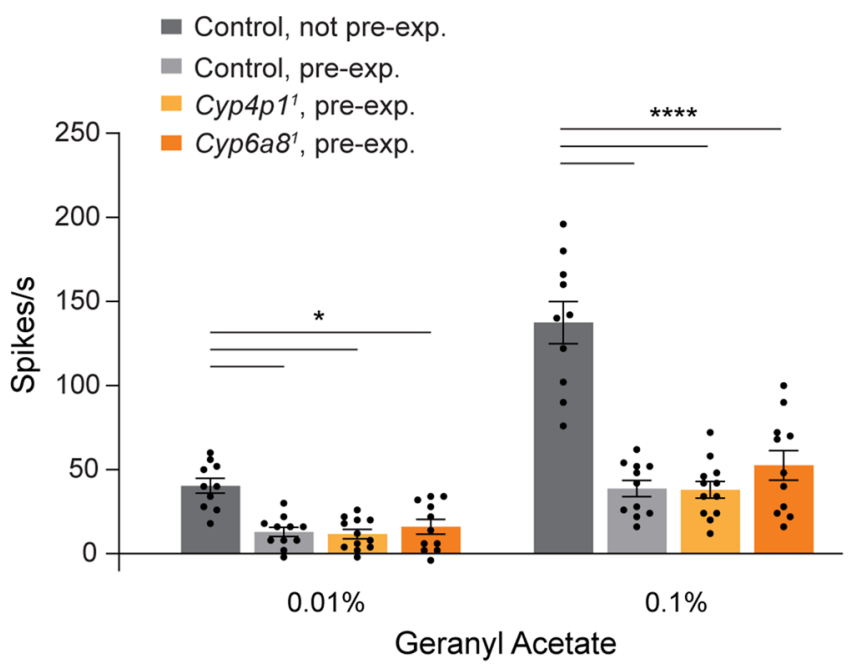

Figure 5. Pre-exposure to geranyl acetate reduces spiking responses to geranyl acetate. (a) SSR traces show responses of an ab5 sensillum to a $500 \mathrm{~ms}$ pulse of $0.01 \%$ and $0.1 \%$ geranyl acetate in control flies not exposed to geranyl acetate. (b) SSR traces show responses of an ab5 sensillum to geranyl acetate in control flies following two hours of exposure to $5 \%$ geranyl acetate. (c,d) Similar to (b), but for (c) Cyp4p $1^{1}$ and (d) Cyp6a $8^{1}$ mutants. (e) Quantification of spiking responses to geranyl acetate. Bar graphs depict the mean \pm SEM overlaid with the individual data points. Data were analyzed with a two-way ANOVA followed by Tukey's post-hoc test. Statistical significance is presented as ${ }^{\star} p<0.05$ and ${ }^{\star * *} p<0.0001$. Other comparisons were not significant $(p>0.05)$.

broadly expressed, there may be some Cyps whose expression is limited to particular sensillar classes, and thus may contribute to olfactory signaling by the neurons housed in those sensilla.

Surprisingly few studies have investigated the localization of CYPs within antennae in either Drosophila or other insects ${ }^{17,41}$. To explore whether CYPs with restricted expression exist in Drosophila, we selected four antennal CYPs for reporter line generation: (1) Cyp4e2, one of the most highly expressed CYPs in the antenna ${ }^{11}$, (2) Cyp308a1, a CYP exclusively found in the antenna ${ }^{12,13}$, and (3) Cyp313a4 and Cyp4d21, whose expression is greatly reduced in atonal mutants which fail to develop coeloconic sensilla, the sacculus and the arista ${ }^{11,42}$. Examination of mtdTomato driven by the CYP reporter lines in the antenna revealed that Cyp308a1 and Cyp4e2 are widely expressed (Fig. 6a,b). In contrast, expression of Cyp313a4 is restricted to a small scattered subset of sensilla (Fig. 6c) and Cyp4d21 expression is limited to the arista (Fig. 6d). We suggest that investigation of Cyp313a4 and other Cyps with restricted sensillar expression may prove useful in future studies on the role of CYPs in olfactory neuron function. 

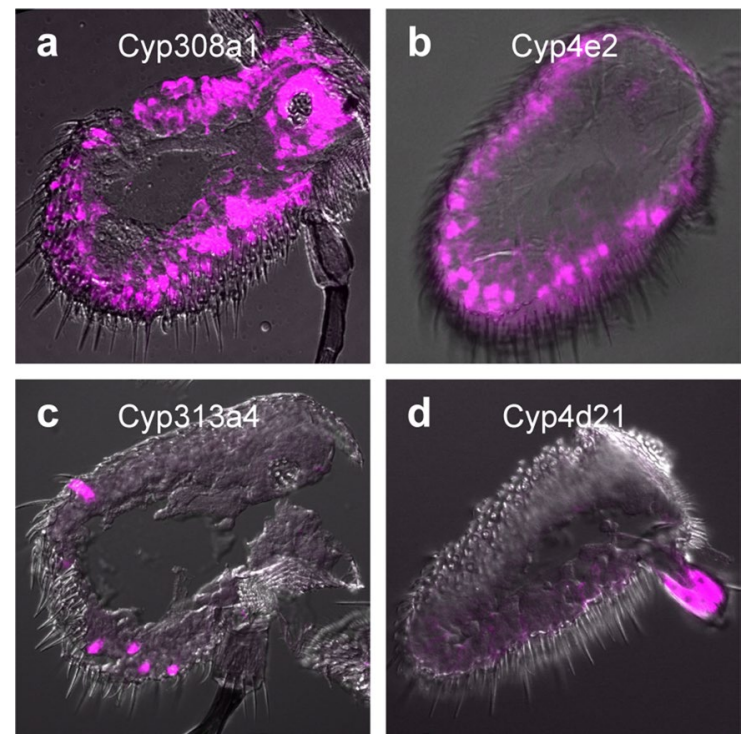

Figure 6. CYPs can have both broad and narrow antennal expression patterns. (a-d) Confocal images of antennal sections labeled with an antibody against mtdTomato (magenta) driven by (a) Cyp308a1-LexA, (b) Cyp4e2-LexA, (c) Cyp313a4-LexA, or (d) Cyp4d21-LexA. Cyp308a1 and Cyp4e2 are broadly expressed, whereas Cyp313a4 is expressed in a subset of sensilla and Cyp4d21 is expressed in the arista.

\section{Discussion}

In this study, we found that exposure to the odorant geranyl acetate specifically and consistently leads to the induction of three CYPs, Cyp4p1, Cyp6a8, and Cyp6d5, in the antenna. Interestingly, each of these CYPs has been previously shown to be inducible by multiple xenobiotics ${ }^{22-25}$. The xenobiotics that upregulate these CYPs vary widely in structure. For example, exposure to phenobarbital, caffeine, atrazine, pyrethrum, paraquat, or piperonyl butoxide lead to the induction of one or more of these CYPs ${ }^{22,25}$. Additionally, each of these three CYPs has a high level of genomic instability, with frequent gene gain and loss events across Drosophila species ${ }^{21}$. These findings, together with the enrichment of Cyp4p1, Cyp6a8, and Cyp6d5 in tissues associated with detoxification such as the midgut, Malpighian tubules and fat body, suggest they function in xenobiotic detoxification rather than the synthesis or degradation of products as part of developmental or physiological processes ${ }^{18}$. This would be consistent with a role for these CYPs in the degradation of odorants such as geranyl acetate.

We also investigated the localization and function of geranyl acetate-induced CYPs in the antenna. Although bulk RNASeq studies demonstrate that many CYPs are expressed in the Drosophila antenna, their localization within this tissue has rarely been examined. Using FISH, we found that Cyp4p1 and Cyp6d5 are broadly expressed within the antenna, suggesting they are found in multiple sensilla classes. Further, our data revealed these CYPs are not expressed by olfactory neurons. We suspect that they are expressed by the non-neuronal auxiliary cells, which have long been thought to mediate odorant degradation ${ }^{2}$.

We generated homozygous mutants for Cyp4p1 and Cyp6a8, and used these for electrophysiological recordings on the geranyl acetate sensitive ab5 sensilla. We probed multiple aspects of geranyl acetate responsiveness, including sensitivity, kinetics, and repetitive stimuli, but did not find any differences between controls and the CYP mutants. We also examined olfactory neuron responses following prolonged exposure ( $2 \mathrm{~h})$ to geranyl acetate, a situation in which the absence of CYPs may enhance odorant accumulation in the sensillum lymph and lead to desensitization. Following geranyl acetate exposure, electrophysiological responses to geranyl acetate were strongly reduced. This is similar to what has been reported for other odorants and is thought to be mediated by de-phosphorylation of the Orco co-receptor ${ }^{43,44}$. However, slow desensitization was not affected by the loss of Cyp4p1 or Cyp6a8.

Our negative results may indicate that odorant degradation mediated by CYPs does not affect olfactory signaling. However, there are multiple caveats that prevent this conclusion. First and foremost, it is unclear whether the induction of a CYP by a xenobiotic compound necessarily implies that the compound is metabolized by that CYP. Although numerous studies demonstrate Cyp4p1, Cyp6a8, or Cyp6d5 induction in Drosophila, to our knowledge only one has directly demonstrated that the xenobiotic is modified or degraded by the induced CYP ${ }^{25}$. More generally, the signal transduction pathway by which xenobiotics induce CYP expression is poorly understood in insects ${ }^{26}$. As a result, it is possible that xenobiotic exposure leads to the activation of transcription factors that increase expression of a broad array of CYPs, not all of which target the xenobiotic. A second caveat is that CYPs may play redundant roles in odorant degradation, such that the absence of one CYP is compensated by another. We tested each mutant alone, but not in combination. Further, it is possible that Cyp $6 d 5$ acts on geranyl acetate, but the lethality of our Cyp $6 d 5^{1}$ mutant prevented us from testing the role of this enzyme. Going forward, the role of this CYP could be tested with an RNAi construct expressed selectively in the antenna. A third caveat is that we only examined CYP induction following exposure to two odorants, neither of which is a preferential CYP substrate such as an aromatic compound. Identifying the CYPs induced by a larger number of odorants 
and testing their function with electrophysiology on genetic mutants will allow for a more robust evaluation of the transcriptional deorphanization approach. Given these caveats, we do not believe our data can rule out a role for CYPs in olfactory signaling.

We studied the three CYPs induced by geranyl acetate as we fortuitously discovered their selective upregulation following exposure to this odorant. This odorant is known to be a substrate for a bacterial CYP ${ }^{32}$. However, other classes of ODEs including esterases, GSTs, and UDP-glucuronosyltransferases (UGTs) are also known to metabolize odorants and affect olfactory signaling ${ }^{1-3}$. Both of the odorants we utilized, geranyl acetate and ethyl lactate, are esters and as such may be degraded by esterases. Indeed, examination of our RNASeq dataset revealed two carboxylesterases, lama and puml, that are upregulated by exposure to geranyl acetate (Supplementary Fig. S2 and Supplementary Data S1). Their upregulation by geranyl acetate exposure may contribute to the reduced spiking responses to geranyl acetate following odorant pre-exposure as seen in Fig. 5. In contrast, no carboxylesterases were upregulated by ethyl lactate. We also examined our RNASeq dataset for other classes of ODEs. Seven GSTs and two UGTs were upregulated by geranyl acetate exposure (Supplementary Fig. S2 and Supplementary Data S1). Together, these data suggest additional candidates for studying whether a transcriptional deorphanization approach is useful for the study of ODEs involved in insect olfaction.

Given the limitations of a transcriptional deorphanization approach, how might the role of CYPs be studied in Drosophila olfaction in the future? We have begun to generate a collection of transgenic CYP reporter lines to investigate their expression patterns within the antenna. We have identified some CYPs with highly restricted expression patterns, such as Cyp313a4. Such CYPs most likely act on a narrow range of substrates rather than fulfilling a general metabolic role, and quite likely these substrates would include odorants detected by that sensillum. We propose that identifying the sensilla classes in which CYPs with restricted expression are found and then testing their role in responses mediated by neurons in those sensilla will be informative in the future.

\section{Methods}

Fly lines. Drosophila melanogaster flies were raised on a standard cornmeal molasses food at $25{ }^{\circ} \mathrm{C}$ in an incubator with a 12:12 h day/night cycle. Canton-S flies were used for RNA sequencing and qRT-PCR. FISH was carried out on lines in which either Ir8a-Gal4 $4^{11}$ or Orco-Gal4 ${ }^{45}$ drove expression of UAS-mCD8::GFP $P^{46}$. LexA lines for Cyp308a1, Cyp4e2, Cyp313a4, and Cyp4d21 were generated as described below and drove expression of LexAop-mtdTomato ${ }^{47}$. $C y p 4 p 1^{1}$ and $C y p 6 a 8^{1}$ loss-of-function mutations, generated as described below, were outcrossed for 10 generations to a white-eyed Canton-S $(w C S)$ line prior to electrophysiological recordings ${ }^{47}$. The $w C S$ line served as a control for all electrophysiological experiments.

Generation of transgenic reporter lines. Transgenic LexA reporter lines for Cyp4d21, Cyp308a1, Cyp4e2, and Cyp313a4 were generated using standard methods, similar to described previously ${ }^{11,47}$. The $5^{\prime}$ and $3^{\prime}$ regions flanking the genes were cloned from the following tiling bacterial artificial chromosomes (BACs) corresponding to the Drosophila melanogaster reference genome: BACR13D17 (Cyp4d21), BACR07J06 (Cyp313a4),

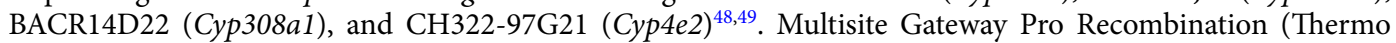
Fisher) was used to assemble the $5^{\prime}$ and $3^{\prime}$ genomic regions surrounding LexA in the pBGRY destination vector ${ }^{47}$. For Cyp4e2-LexA, the $5^{\prime}$ region included chromosome 2R: 8,442,589-8,444,770 and the 3' region 2R: 8,446,8958,447,622. For Cyp4d21-LexA, the 5' region included chromosome 2L: 7,597,215-7,605,812 and the $3^{\prime}$ region 2L: 7,607,235-7,610,142. For Cyp313a4-LexA, the 5' region included chromosome 3R: 12,681,257-12,683,325 and the $3^{\prime}$ region 3R: 12,678,913-12,679,412. For Cyp308a1-LexA, the $5^{\prime}$ region included chromosome X: $18,705,774-18,709,894$ and the $3^{\prime}$ region X: 18,711,500-18,716,181. Plasmids were verified with restrictions digests and sequencing. The vectors were injected Drosophila embryos by BestGene, Inc. for PhiC31-mediated genomic integration ${ }^{50}$. Vectors were integrated into either attP40 (2nd chromosome, Cyp4e2-LexA, Cyp4d21LexA, and Cyp308a1), VK27 attP-9A (3rd chromosome, Cyp4e2-LexA and Cyp4d21-LexA), or attP2 (3rd chromosome, Cyp313a4-LexA) landing sites ${ }^{51-53}$. Flies with successful integration were identified by expression of mini-white in their eyes.

Generation of CYP mutants. Cyp4p $1^{1}, C y p 6 a 8^{1}$, and $C y p 6 d 5^{1}$ mutant fly lines were generated with CRISPR/Cas 9 engineering and homology directed repair, similar to described previously ${ }^{54}$. Of the coding sequences for each gene, $94 \%$ are deleted in $C y p 4 p 1^{1}, 35 \%$ for $C y p 6 a 8^{1}$, and $54 \%$ for $C y p 6 d 5^{1}$. For each gene, guide RNA (gRNA) sequences were inserted into pU6-BbsI-chiRNA ${ }^{55}$ using the Q5 site-Directed Mutagenesis Kit (New England Biolabs). Each PCR used a common primer GAAGTATTGAGGAAAACATA and a primer containing the gene specific gRNA sequence followed by GTTTTAGAGCTAGAAATAGC. Correct guide-RNA plasmids were verified by sequencing. For each gene, one or two gRNA sequences were generated with the following primers: GCCAGTAGAGCAGGGCACTT and GCAGAAGATTGTCTTCCATA for Cyp4p1, GGT TAGCTCGTATAGGGTAA for Cyp6d5, and GAAAGACGGAATCTATCTGA and GATTCTTTGCCAGTT CGTAT for Cyp6a8. Donor plasmids for homology directed repair were generated by cloning homology arms from Canton-S genomic DNA with Phusion Hot Start DNA polymerase (New England Biolabs) and inserting them sequentially into the pHD-DsRed-attP vector using restriction enzymes ${ }^{56}$. The homology arms flanked the $3 \mathrm{xP} 3$-DsRed marker after being inserted into the vector. The homology arms for Cyp4p1 spanned from chromosome 2R: 9,239,359-9,240,112 and 2R: 9,241,981-9,242,939. The homology arms for Cyp6a8 spanned from chromosome 2R: 14,886,540-14,887,420 and 2R: 14,888,010-14,888,943. The homology arms for Cyp6d5 spanned from chromosome 3R: 14,028,829-14,029,440 and 3R: 14,030,647-14,031,375. Donor plasmids and gRNA plasmids were injected into embryos by BestGene, Inc. The injected embryo lines were $y$, w;;nos-Cas9(IIIattP2) for Cyp4p1 and Cyp6a8 and y,w;nos-Cas9(II-attP40) for Cyp6 $\mathrm{d}^{57}$. 
Ocular DsRed expression was used to identify flies in which the donor plasmid had been integrated into the genome. Sequencing of PCR products was used to validate the expected genetic changes. Homozygous flies were obtained for mutations in Cyp4p1 and Cyp6a8 mutations, but not for Cyp6d5. Flies were outcrossed for 10 generations to the $w C S$ genetic background prior to experiments.

Antennal immunohistochemistry. Flies aged approximately one week were anesthetized, aligned in a collar, and encased in OCT (Tissue-Tek) up to their necks in a silicone mold. Following freezing on dry ice, heads were snapped off and stored as blocks at $-80^{\circ} \mathrm{C}$. Sections $(20 \mu \mathrm{m})$ were collected on a Leica cryostat and used for immunohistochemistry. Slides were fixed in $4 \%$ formaldehyde in PBS for $10 \mathrm{~min}$, washed three times in PBS, permeabilized in PBS with $0.1 \%$ Tween (PBST) for $30 \mathrm{~min}$, and then immersed in blocking buffer (PBST with $1 \%$ BSA) for $30 \mathrm{~min}$. Sections were incubated overnight at $4{ }^{\circ} \mathrm{C}$ in rabbit anti-RFP antibody (Rockland, RRID:AB_2209751) diluted 1:500 in blocking buffer. Following removal of excess primary antibody by three washes in PBST, the secondary antibody goat anti-rabbit (Fisher, RRID:AB_143157) was applied at 1:500 diluted in blocking buffer for two hours at room temperature. Sections were then washed three times in PBST and mounted in Vectashield. Imaging was performed with a Nikon A1R confocal microscope at Advanced Light Microscopy Facility at the University of Connecticut. Images were process with ImageJ/FIJI software.

RNA sequencing. Canton-S flies $(\sim 100)$ aged 7-10 days were exposed for $5 \mathrm{~h}$ to $5 \%$ geranyl acetate in DMSO, 5\% ethyl lactate in DMSO, or $20 \mu \mathrm{l}$ DMSO. Exposures took place in a $25^{\circ} \mathrm{C}$ incubator in narrow plastic fly vials containing fresh food and a $13 \mathrm{~mm}$ GE Whatman antibiotic disc with $20 \mu \mathrm{l}$ of the odorant solution. Exposure vials were kept in $500 \mathrm{ml} \mathrm{screw}$-top containers to prevent mixing of odors. After $5 \mathrm{~h}$, vials were placed at $-20{ }^{\circ} \mathrm{C}$ until flies were immobilized. Flies were flash frozen in liquid nitrogen, and the antennae were manually dissected into a $1.5 \mathrm{ml}$ Eppendorf tube set in a liquid nitrogen bath. After collection of $\sim 200$ antennae, the antennae were pelleted by centrifugation at $4{ }^{\circ} \mathrm{C}$. Three independent antennal samples were collected for each odorant condition. Antennal tissues were ground using an RNAse-free pestle, and total RNA was extracted using QIAShredders (Qiagen) and the QIAGEN RNeasy Micro kit. The RNeasy Micro kit protocol was followed as directed. The RNA was then cleared of genomic DNA using DNAse from an iScript gDNA Clear cDNA Synthesis Kit (Bio-Rad).

The nine RNA samples $(\sim 0.5 \mu \mathrm{g}$ each) were sent to the Center for Genome Innovation at the University of Connecticut for quality control, library preparation, and sequencing. Libraries were prepared using the Illumina mRNA sample prep kit for non-stranded RNA, and sequencing was carried out on an Illumina NextSeq 500 with a mid output 150 cycle sequencing run to produce paired-end reads.

RNA sequencing analysis. Raw reads were obtained from BaseSpace Sequence Hub (Illumina). Sickle was used with the default parameters to perform quality control and produce trimmed reads ${ }^{58}$. STAR was used with the default parameters to align trimmed reads to the Drosophila melanogaster reference genome (dm6 2014, Release 6 plus ISO1 MT) with mitochondrial genes removed ${ }^{59,60}$. Raw counts of reads mapping uniquely to each gene were obtained using HTSeq-count with the "intersection-nonempty" and "nonunique-none" modes ${ }^{61}$. Mapped reads are available at the NCBI SRA under bioProject accession number PRJNA746306.

The Reads per Million mapped reads (RPM) for each gene was calculated by dividing the raw reads by total reads, then multiplying by $1,000,000$. We identified known antennal IR and OR olfactory receptors within the dataset, and these are shown in Supplementary Data S1. The 87 CYPs in the Drosophila genome were identified within the dataset, and the 42 CYPs whose expression was > 5 RPM in each DMSO-exposed sample were considered to be antennal-expressed (Supplementary Data S1). We similarly extracted information for antennalexpressed ODEs including GSTs, UGTs, and carboxylesterases for gene groups identified through FlyBase ${ }^{62}$. For carboxylesterases, we focused on the 37 "other carboxylesterases" group, some of which are linked to xenobiotic metabolism (Supplementary Data S1). For each dataset, an ExactTest was run in EdgeR v3.26.8 ${ }^{63}$ in $\mathrm{R}$ v3.6.1 to identify differentially expressed genes between flies exposed to geranyl acetate or to DMSO. Separate paired ExactTests were run for ethyl lactate and DMSO, and ethyl lactate and geranyl acetate. The 42 antennal-expressed CYPs were extracted from each analysis. Of these, five CYPs were differentially expressed with FDR $<0.15$ between flies exposed to geranyl acetate and those exposed to DMSO (Supplementary Data S1). These CYPs were also differentially expressed between flies exposed to geranyl acetate and those exposed to ethyl lactate. Of these, Cyp6a8, Cyp6d5, and Cyp4p1 were upregulated by geranyl acetate exposure, whereas Cyp313a1 and Cyp6v1 were downregulated. No CYPs were differentially expressed between flies exposed to ethyl lactate and those exposed to DMSO. Olfactory receptors, GSTs, UGTs, and carboxylesterases were also extracted from the ExactTests and are included in Supplementary Data S1.

qRT-PCR analysis. Canton-S flies $(\sim 100)$ aged 7-10 days were exposed to $20 \mu \mathrm{l}$ of $5 \%$ geranyl acetate in DMSO, 5\% ethyl lactate in DMSO, or DMSO, in the same manner as for the RNASeq experiment. Flies were exposed for two hours, except when examining the time course of the transcriptional response when they were exposed for 0 to $12 \mathrm{~h}$. Odorant exposure and total RNA isolation was carried out as described above. Total RNA (280 ng per sample) was used to generate cDNA using the iScript gDNA Clear cDNA Synthesis Kit (Bio-Rad). The cDNA was used as a template for qRT-PCR with SsoFast EvaGreen Supermix (Bio-Rad). A CFX96 thermocycler (Bio-Rad) was used to run the qRT-PCR assays in triplicate. For all experiments, gene expression in each sample was measured relative to the housekeeping gene eIF1a to normalize cDNA levels between samples. For the time course analysis, expression at each time point is plotted as the fold change relative to expression at $0 \mathrm{~h}$ of geranyl acetate exposure. For the dose response analysis, expression at each geranyl acetate concentration 
is plotted as the fold change relative to exposure to DMSO alone. Each reaction was run on 4-6 independent antennal replicates.

Probe synthesis for fluorescence in situ hybridization (FISH). The Cyp4p1 gene was amplified from the Canton-S antennal cDNA with primers ATGATTATCTTGTGGCTGATTCTG and AATAAGTCACGTTCG CCTCAC and then ligated into pGEM-T Easy (Promega). The vector carrying the full length Cyp4p1 insert was verified by restriction digests and sequencing. Plasmids containing full length cDNA sequences for Cyp $6 d 5$ and Cyp6a8 in the pOT2 vector, GH07481 and FI17852 respectively ${ }^{64}$, were obtained from the Drosophila Genomics Resource Center.

To generate FISH sense (S) and anti-sense (AS) probes, 1-5 $\mu$ g of Cyp4p1-pGEM, Cyp6a8-pOT2 and Cyp6d5pOT2 plasmids were linearized overnight and purified into RNAse-free water using the MinElute Reaction Cleanup Kit (Qiagen). Labeled S and AS probes were generated using the DIG RNA Labeling Kit (SP6/T7) (Roche) per the manufacturer's instructions. Probes were hydrolyzed for $10 \mathrm{~min}$ in $30 \mathrm{mM} \mathrm{Na}_{2} \mathrm{CO}_{3}, 20 \mathrm{mM}$ $\mathrm{NaHCO}_{3}(\mathrm{pH}$ 10.2). The reaction was terminated with $3 \mathrm{M} \mathrm{NaOAc}, 1 \%$ acetic acid ( $\mathrm{pH} 6$ ). Probes were then purified with ethanol precipitation, solubilized and diluted to $50 \mathrm{ng} / \mu \mathrm{L}$ in DEPC water, and stored at $-80^{\circ} \mathrm{C}$.

Combined FISH and immunocytochemistry. Groups of 4-7-day old flies were exposed to geranyl acetate or DMSO for two hours. After exposure, flies were anesthetized by cold, maintained on a petri dish on an ice bath, and manually placed on a collar. The heads of 5 males and 5 females were covered with silicone molds containing OCT (Tissue-Tek) and placed on dry ice, and the blocks were snapped off when frozen. The blocks were sectioned at $20 \mu \mathrm{m}$ using a cryostat. The slides were fixed with $4 \%$ paraformaldehyde in PBS for $10 \mathrm{~min}$, washed with PBS, and then incubated for $10 \mathrm{~min}$ in an acetylation solution $(0.463 \mathrm{~g}$ triethanolamine $\mathrm{HCl}, 56$ $\mu \mathrm{L} 10 \mathrm{~N} \mathrm{NaOH}, 62.5 \mu \mathrm{L}$ acetic anhydride, $25 \mathrm{~mL} \mathrm{H} 20$ ). Slides were then washed in PBS and prehybridized for one hour at $65^{\circ} \mathrm{C}$ in hybridization buffer (HB: $25 \mathrm{~mL}$ formamide, $12.5 \mathrm{ml} 20 \times$ SSC, $2.5 \mathrm{mg}$ heparin, $0.5 \mathrm{~mL} 10 \%$ Tween-20, $12 \mathrm{~mL} \mathrm{DEPC} \mathrm{H}_{2}$ ) . DIG labeled probes were diluted to $500 \mathrm{ng} / \mathrm{mL}$ in $\mathrm{HB}$, denatured at $80{ }^{\circ} \mathrm{C}$ for $5 \mathrm{~min}$, and cooled on ice for one minute. Slides were covered with $200 \mu \mathrm{L}$ of probe in $\mathrm{HB}$ and a Hybrislip (SigmaAldrich). They were then incubated at $65^{\circ} \mathrm{C}$ in a humid chamber for $\sim 20 \mathrm{~h}$. Hybrislips were removed by dunking in $5 \times \mathrm{SSC}$ warmed to $65^{\circ} \mathrm{C}$, and they were then washed three times in $0.2 \times \mathrm{SSC}$ at $65^{\circ} \mathrm{C}$. Slides were then incu-

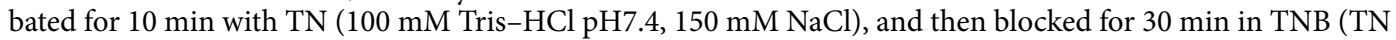
with $1 \%$ Blocking Reagent (Roche)) at room temperature. Primary antibody solution containing anti-DIG-POD antibody (1:250, Roche, RRID:AB_514500) and mouse anti-GFP antibody (1:500, Roche, RRID:AB_390913) diluted in TNB was added to each slide under a bridged coverslip and incubated at $4{ }^{\circ} \mathrm{C}$ overnight. After careful removal of the bridged coverslips, slides were washed in TNT (TN with 0.05\% Tween-20). TSA-Cy3 was diluted 1:50 in Amplification Reagent (Perkin-Elmer), and each slide was incubated with $200 \mu \mathrm{L}$ of this solution in the dark for $10 \mathrm{~min}$ at room temperature. All further steps were carried out in the dark. Slides were washed in TNT with agitation, and then incubated for $2 \mathrm{~h}$ at room temperature in Alexa Fluor 488 donkey-anti-mouse (1:500 in TNB, Invitrogen, RRID:AB_141607). Following three washes with TNT, and sections were mounted in Vectashield (Vector Labs). Confocal microscopy was performed using a Nikon A1R Confocal microscope in the University of Connecticut Advanced Light Microscopy Facility. All images were processed using FIJI/Image ${ }^{65}$.

Odorants. Odorants were geranyl acetate (95\%, Alpha Aesar), ethyl lactate (99\%, Alpha Aesar), and citral (95\%, Sigma). Geranyl acetate and ethyl lactate were diluted into DMSO (Fisher Chemical) for exposing flies prior to RNASeq, qRT-PCR, FISH histology, and electrophysiology. They were used at a 5\% concentration, except for the dose-response curve shown in Fig. 1d. For electrophysiological recordings, geranyl acetate and ethyl lactate were diluted 1:10 into paraffin oil (Sigma), and serial dilutions were used to achieve lower concentrations. In Fig. 3a, geranyl acetate was used at $0.1 \%$ and citral at $0.1 \%$. In Figs. $3 c$ and 4 , geranyl acetate was used at $0.01 \%$.

Electrophysiology. Single sensillum electrophysiological recordings were performed on 3-7 days old female flies as described ${ }^{66,67}$. In brief, flies were wedged in a $200 \mu$ pipette tip, exposing a portion of their head and their antennae. One antenna was held gently between a tapered glass electrode and a coverslip. The prep was placed on BX51WI microscope (Olympus) under a continuous 2,000 $\mathrm{mL} / \mathrm{min}$ humidified air stream delivered through the glass main airflow tube. Borosilicate glass electrodes were filled with the sensillum lymph ringers solution ${ }^{68}$ were inserted into the eye and individual sensilla as reference and recording electrodes, respectively. The ab5 sensilla were distinguished by their location on the antenna and their response to a small number of diagnostic odorants. No more than 3 sensilla were recorded per fly. Extracellular action potential recordings were determined with an EXT-02B amplifier (NPI) with a $5 \times$ gain headstage. Data were obtained and AC filtered $(300-3,000 \mathrm{~Hz})$ at $10 \mathrm{kHz}$ with a PowerLab 4/35 digitizer and Lab Chart Pro v8 software (ADInstruments).

Odorant cartridges consisted of $14.6 \mathrm{~cm}$ Pasteur pipettes capped with $1 \mathrm{~mL}$ pipette tips. Prior to capping, $50 \mu \mathrm{l}$ of odorant solution was pipetted onto a $13 \mathrm{~mm}$ antibiotic assay disc (Whatman) and inserted into each Pasteur pipette. Odorants were allowed to equilibrate for at least $30 \mathrm{~min}$. To apply the odorants, air at $500 \mathrm{~mL} /$ min flowed for $500 \mathrm{~ms}$ through the cartridge, which was inserted into a hole in the main airflow tube. Odorant delivery was controlled by LabChart, which directed the opening of a valve linked to a ValveBank 4 controller (Automate Scientific). Cartridges were used up to 4 times, with at least $10 \mathrm{~min}$ recovery between trials on different sensilla. A $10 \mathrm{~s}$ trace was collected, including a $1 \mathrm{~s}$ baseline prior to odorant application. Each sensillum was tested with multiple odorants with at least $10 \mathrm{~s}$ between odorant applications.

For recordings in Fig. 4, an ab5 sensillum was first identified with the standard recording setup described above. Then, paired pulses and trains of geranyl acetate stimuli were applied to the sensillum with a modified 
delivery system. For this, a $1000 \mathrm{~mL}$ Erlenmeyer flask was filled with $200 \mathrm{~mL}$ of $0.01 \%$ geranyl acetate. The flask was capped using a rubber stopper with 2 openings. Through one opening, an input line was inserted. The other opening contained the output line that connected to the main airflow tube. A Lee valve upstream of the input line was used to divert a stream of $500 \mathrm{ml} / \mathrm{min}$ air through the geranyl acetate flask and into the main airflow tube. LabChart was programmed to change odorant delivery systems and to apply the paired pulse and spike train stimuli. The three paired pulse stimuli were obtained were applied in a continuous recording by applying $500 \mathrm{~ms}$ of geranyl acetate at 1,11,26,29,44, and $45.5 \mathrm{~s}$. Spike trains were obtained by applying $200 \mathrm{~ms}$ of geranyl acetate every second for five seconds.

Action potentials were detected offline using LabChart Spike Histogram software. Spikes from ab5A and ab5B neurons were summed due to their similar amplitudes ${ }^{69}$. Action potentials were counted over a $500 \mathrm{~ms}$ window, $100 \mathrm{~ms}$ after stimulus onset to account for the line delay. Solvent corrected odorant responses graphed in Figs. 3a,b, and 5 were determined as the number of spikes induced by the odorant after subtracting the number of spikes produced by stimulating with paraffin oil alone. In Fig. 4d, solvent corrected responses were used to calculate paired-pulse ratios as Response ${ }_{2} /$ Response $_{1}$. In Fig. 4f, solvent corrected responses were normalized by dividing each response by the mean response to the first pulse of geranyl acetate for that genotype. For the peristimulus time histograms (PSTHs) in Fig. 3c, spikes were identified in LabChart with the Spike Histogram feature, and spike timing data were exported to Igor Pro 8.04 (WaveMetrics) where responses were averaged in $50 \mathrm{~ms}$ bins to generate PSTHs for each sensillum. PSTHs were then averaged to obtain the mean \pm SEM PSTHs.

Received: 6 August 2021; Accepted: 4 October 2021

Published online: 15 October 2021

\section{References}

1. Leal, W. S. Odorant reception in insects: Roles of receptors, binding proteins, and degrading enzymes. Annu. Rev. Entomol. 58, 373-391 (2013).

2. Vogt, R. G. Molecular basis of pheromone detection in insects. In Comprehensive Insect Physiology, Biochemistry, Pharmacology and Molecular Biology, Vol. 3 Endocrinology (eds Gilbert, L. I. et al.) 753-804 (Elsevier, 2005).

3. Heydel, J. M., Faure, P. \& Neiers, F. Nasal odorant metabolism: Enzymes, activity and function in olfaction. Drug Metab. Rev. 51, 224-245 (2019).

4. Maibeche-Coisne, M., Nikonov, A. A., Ishida, Y., Jacquin-Joly, E. \& Leal, W. S. Pheromone anosmia in a scarab beetle induced by in vivo inhibition of a pheromone-degrading enzyme. Proc. Natl. Acad. Sci. 101, 11459-11464 (2004).

5. Thiebaud, N. et al. Odorant metabolism catalyzed by olfactory mucosal enzymes influences peripheral olfactory responses in rats. PLoS ONE 8, e59547 (2013).

6. Nagashima, A. \& Touhara, K. Enzymatic conversion of odorants in nasal mucus affects olfactory glomerular activation patterns and odor perception. J. Neurosci. 30, 16391-16398 (2010).

7. Feyereisen, R. Insect CYP genes and P450 enzymes. In Insect Molecular Biology and Biochemistry (ed. Gilbert, L.) 236-316 (Elsevier, 2012).

8. Tijet, N., Helvig, C. \& Feyereisen, R. The cytochrome P450 gene superfamily in Drosophila melanogaster: Annotation, intron-exon organization and phylogeny. Gene 262, 189-198 (2001).

9. Dermauw, W., Van Leeuwen, T. \& Feyereisen, R. Diversity and evolution of the P450 family in arthropods. Insect Biochem. Mol. Biol. 127, 103490 (2020).

10. Chung, H. et al. Characterization of Drosophila melanogaster cytochrome P450 genes. Proc. Natl. Acad. Sci. 106, 5731-5736 (2009).

11. Menuz, K., Larter, N. K., Park, J. \& Carlson, J. R. An RNA-seq screen of the Drosophila antenna identifies a transporter necessary for ammonia detection. PLoS Genet. 10, e1004810 (2014).

12. Younus, F. et al. Identification of candidate odorant degrading gene/enzyme systems in the antennal transcriptome of Drosophila melanogaster. Insect Biochem. Mol. Biol. 53, 30-43 (2014).

13. Mohapatra, P. \& Menuz, K. Molecular profiling of the Drosophila antenna reveals conserved genes underlying olfaction in insects. G3 (Bethesda) 9, 3753-3771 (2019).

14. Wang, Q., Hasan, G. \& Pikielny, C. W. Preferential expression of biotransformation enzymes in the olfactory organs of Drosophila melanogaster, the antennae. J. Biol. Chem. 274, 10309-10315 (1999).

15. Wojtasek, H. \& Leal, W. S. Degradation of an alkaloid pheromone from the pale-brown chafer, Phyllopertha diversa (Coleoptera: Scarabaeidae), by an insect olfactory cytochrome P450. FEBS Lett. 458, 333-336 (1999).

16. Keeling, C. I. et al. CYP345E2, an antenna-specific cytochrome P450 from the mountain pine beetle, Dendroctonus ponderosae Hopkins, catalyses the oxidation of pine host monoterpene volatiles. Insect Biochem. Mol. Biol. 43, 1142-1151 (2013).

17. Feng, B., Zheng, K., Li, C., Guo, Q. \& Du, Y. A cytochrome P450 gene plays a role in the recognition of sex pheromones in the tobacco cutworm, Spodoptera litura. Insect Mol. Biol. 26, 369-382 (2017).

18. Scanlan, J. L., Gledhill-Smith, R. S., Battlay, P. \& Robin, C. Genomic and transcriptomic analyses in Drosophila suggest that the ecdysteroid kinase-like (EcKL) gene family encodes the "detoxification-by-phosphorylation" enzymes of insects. Insect Biochem. Mol. Biol. 123, 103429 (2020).

19. Nauen, R., Zimmer, C. T. \& Vontas, J. Heterologous expression of insect P450 enzymes that metabolize xenobiotics. Curr. Opin. Insect Sci. 43, 78-84 (2021).

20. Schuler, M. A. \& Berenbaum, M. R. Structure and function of cytochrome P450S in insect adaptation to natural and synthetic toxins: Insights gained from molecular modeling. J. Chem. Ecol. 39, 1232-1245 (2013).

21. Good, R. T. et al. The molecular evolution of cytochrome P450 genes within and between drosophila species. Genome Biol. Evol. 6, 1118-1134 (2014).

22. Giraudo, M., Unnithan, G. C., Le Goff, G. \& Feyereisen, R. Regulation of cytochrome P450 expression in Drosophila: Genomic insights. Pestic. Biochem. Physiol. 97, 115-122 (2010).

23. Willoughby, L. et al. A comparison of Drosophila melanogaster detoxification gene induction responses for six insecticides, caffeine and phenobarbital. Insect Biochem. Mol. Biol. 36, 934-942 (2006).

24. Misra, J. R., Horner, M. A., Lam, G. \& Thummel, C. S. Transcriptional regulation of xenobiotic detoxification in Drosophila. Genes Dev. 25, 1796-1806 (2011).

25. Coelho, A. et al. Cytochrome P450-dependent metabolism of caffeine in Drosophila melanogaster. PLoS ONE 10, e0117328 (2015).

26. Li, X., Deng, Z. \& Chen, X. Regulation of insect P450s in response to phytochemicals. Curr. Opin. Insect Sci. 43, 108-116 (2021).

27. Wu, C. et al. Exposure of Helicoverpa armigera Larvae to plant volatile organic compounds induces cytochrome P450 monooxygenases and enhances larval tolerance to the insecticide methomyl. Insects 12, 238 (2021). 
28. Wu, H. et al. Transcriptome analysis of antennal cytochrome P450s and their transcriptional responses to plant and locust volatiles in Locusta migratoria. Int. J. Biol. Macromol. 149, 741-753 (2020).

29. Cano-Ramirez, C. et al. Isolation and expression of cytochrome P450 genes in the antennae and gut of pine beetle Dendroctonus rhizophagus (Curculionidae: Scolytinae) following exposure to host monoterpenes. Gene 520, 47-63 (2013).

30. Lopez, M. F., Cano-Ramirez, C., Cesar-Ayala, A. K., Ruiz, E. A. \& Zuniga, G. Diversity and expression of P450 genes from Dendroctonus valens LeConte (Curculionidae: Scolytinae) in response to different kairomones. Insect Biochem. Mol. Biol. 43, 417-432 (2013).

31. von der Weid, B. et al. Large-scale transcriptional profiling of chemosensory neurons identifies receptor-ligand pairs in vivo. Nat. Neurosci. 18, 1455-1463 (2015).

32. Child, S. A., Naumann, E. F., Bruning, J. B. \& Bell, S. G. Structural and functional characterisation of the cytochrome P450 enzyme CYP268A2 from Mycobacterium marinum. Biochem. J. 475, 705-722 (2018).

33. Hallem, E. A. \& Carlson, J. R. Coding of odors by a receptor repertoire. Cell 125, 143-160 (2006).

34. Silbering, A. F. et al. Complementary function and integrated wiring of the evolutionarily distinct Drosophila olfactory subsystems. J. Neurosci. 31, 13357-13375 (2011).

35. Marshall, B., Warr, C. G. \& de Bruyne, M. Detection of volatile indicators of illicit substances by the olfactory receptors of Drosophila melanogaster. Chem. Senses 35, 613-625 (2010).

36. Hallem, E. A., Ho, M. G. \& Carlson, J. R. The molecular basis of odor coding in the Drosophila antenna. Cell 117, 965-979 (2004).

37. Task, D. et al. Chemoreceptor co-expression in Drosophila olfactory neurons. Preprint at https://doi.org/10.1101/2020.11.07.35565 1v2 (2021)

38. Larsson, M. C. et al. Or83b encodes a broadly expressed odorant receptor essential for Drosophila olfaction. Neuron 43, 703-714 (2004).

39. Werck-Reichhart, D. \& Feyereisen, R. Cytochromes P450: a success story. Genome Biol. 1, REVIEWS3003.3001 (2000).

40. Kaissling, K. E. Kinetics of olfactory responses might largely depend on the odorant-receptor interaction and the odorant deactivation postulated for flux detectors. J. Comp Physiol. A Neuroethol. Sens. Neural Behav. Physiol. 199, 879-896 (2013).

41. Maibeche-Coisne, M., Jacquin-Joly, E., Francois, M. C. \& Nagnan-Le Meillour, P. cDNA cloning of biotransformation enzymes belonging to the cytochrome P450 family in the antennae of the noctuid moth Mamestra brassicae. Insect Mol. Biol. 11, 273-281 (2002).

42. Gupta, B. P. \& Rodrigues, V. Atonal is a proneural gene for a subset of olfactory sense organs in Drosophila. Genes Cells 2, 225-233 (1997).

43. Guo, H., Kunwar, K. \& Smith, D. Odorant receptor sensitivity modulation in Drosophila. J. Neurosci. 37, 9465-9473 (2017).

44. Poudel, S., Guo, H. \& Smith, D. P. PKC98E regulates odorant responses in Drosophila melanogaster. J. Neurosci. 41, 3948-3957 (2021).

45. Wang, J. W., Wong, A. M., Flores, J., Vosshall, L. B. \& Axel, R. Two-photon calcium imaging reveals an odor-evoked map of activity in the fly brain. Cell 112, 271-282 (2003).

46. Lee, T. \& Luo, L. Mosaic analysis with a repressible cell marker for studies of gene function in neuronal morphogenesis. Neuron 22, 451-461 (1999).

47. Koh, T. W. et al. The Drosophila IR20a clade of ionotropic receptors are candidate taste and pheromone receptors. Neuron 83, $850-865$ (2014).

48. Hoskins, R. A. et al. A BAC-based physical map of the major autosomes of Drosophila melanogaster. Science 287, 2271-2274 (2000).

49. Venken, K. J. et al. Versatile P [acman] BAC libraries for transgenesis studies in Drosophila melanogaster. Nat. Methods 6, 431-434 (2009).

50. Bischof, J., Maeda, R. K., Hediger, M., Karch, F. \& Basler, K. An optimized transgenesis system for Drosophila using germ-linespecific phiC31 integrases. Proc. Natl. Acad. Sci. 104, 3312-3317 (2007).

51. Venken, K. J., He, Y., Hoskins, R. A. \& Bellen, H. J. P[acman]: A BAC transgenic platform for targeted insertion of large DNA fragments in D. melanogaster. Science 314, 1747-1751 (2006).

52. Markstein, M., Pitsouli, C., Villalta, C., Celniker, S. E. \& Perrimon, N. Exploiting position effects and the gypsy retrovirus insulator to engineer precisely expressed transgenes. Nat. Genet. 40, 476-483 (2008).

53. Groth, A. C., Fish, M., Nusse, R. \& Calos, M. P. Construction of transgenic Drosophila by using the site-specific integrase from phage phiC31. Genetics 166, 1775-1782 (2004).

54. Delventhal, R. et al. The taste response to ammonia in Drosophila. Sci. Rep. 7, 43754 (2017).

55. Gratz, S. J. et al. Genome engineering of Drosophila with the CRISPR RNA-guided Cas9 nuclease. Genetics 194, 1029-1035 (2013).

56. Gratz, S. J. et al. Highly specific and efficient CRISPR/Cas9-catalyzed homology-directed repair in Drosophila. Genetics 196, 961-971 (2014).

57. Kondo, S. \& Ueda, R. Highly improved gene targeting by germline-specific Cas9 expression in Drosophila. Genetics 195, 715-721 (2013).

58. Sickle: A sliding-window, adaptive, quality-based trimming tool for FastQ files. v1.33 https://github.com/najoshi/sickle.

59. Dobin, A. et al. STAR: ultrafast universal RNA-seq aligner. Bioinformatics 29, 15-21 (2013).

60. dos Santos, G. et al. FlyBase: introduction of the Drosophila melanogaster Release 6 reference genome assembly and large-scale migration of genome annotations. Nucleic Acids Res. 43, D690-D697 (2014).

61. Anders, S., Pyl, P. T. \& Huber, W. HTSeq-a Python framework to work with high-throughput sequencing data. Bioinformatics 31, 166-169 (2015).

62. Thurmond, J. et al. FlyBase 2.0: The next generation. Nucleic Acids Res. 47, D759-D765 (2019).

63. Robinson, M. D., McCarthy, D. J. \& Smyth, G. K. edgeR: a Bioconductor package for differential expression analysis of digital gene expression data. Bioinformatics 26, 139-140 (2010).

64. Rubin, G. M. et al. A Drosophila complementary DNA resource. Science 287, 2222-2224 (2000).

65. Schindelin, J. et al. Fiji: An open-source platform for biological-image analysis. Nat. Methods 9, 676-682 (2012).

66. Dobritsa, A. A., van der Goes van Naters, W., Warr, C. G., Steinbrecht, R. A. \& Carlson, J. R. Integrating the molecular and cellular basis of odor coding in the Drosophila antenna. Neuron 37, 827-841 (2003).

67. Benton, R. \& Dahanukar, A. Electrophysiological recording from Drosophila olfactory sensilla. Cold Spring Harb. Prot. 2011, 824-838 (2011).

68. Kaissling, K.-E. Single unit and electroantennogram recordings in insect olfactory organs. In Experimental Cell Biology of Taste and Olfaction: Current Techniques and Protocols (eds Spielman, A. I. \& Brand, J. G.) 361-377 (CRC Press, 1995).

69. Zhang, Y. et al. Asymmetric ephaptic inhibition between compartmentalized olfactory receptor neurons. Nat. Commun. 10, 1560 (2019).

\section{Acknowledgements}

We thank John Carlson and the Bloomington Drosophila Stock Center (NIH P40OD018537) for fly lines used in this study. The pBGRY plasmid and LexA::VP16 entry clone were gifts from John Carlson and Tong-Wey Koh. The pU6-BbsI-chiRNA plasmid was a gift from Melissa Harrison, Kate O'Conor-Giles, and Jill Wildonger. 
The pHD-DsRed plasmid was gift from Kate O'Connor-Giles. We thank the Drosophila Genomics Resource Center (NIH grant P40OD010949) for cDNA clones. We appreciate assistance from Olivia Durham and Alina Vulpe for cloning constructs for the Cyp308a1-LexA line. We thank Anastasios Tzingounis for comments on the manuscript. Research in K.M.s laboratory was supported by NIH awards R03DC015629 and R35GM133209. D.A. was supported by NSF REU Award to the University of Connecticut 1852486.

\section{Author contributions}

K.M. conceived the project. RNASeq was performed and analyzed by S.B., P.M., and K.M. The qRT-PCR experiments were performed by S.B. Histology was performed by S.B., H.D., R.S., M.N., and D.A. The mutant and transgenic flies were generated by S.B., R.S., and M.N. All electrophysiological recordings were performed by P.M. The manuscript was written by K.M. with input from all authors.

\section{Competing interests}

The authors declare no competing interests.

\section{Additional information}

Supplementary Information The online version contains supplementary material available at https://doi.org/ 10.1038/s41598-021-99910-9.

Correspondence and requests for materials should be addressed to K.M.

Reprints and permissions information is available at www.nature.com/reprints.

Publisher's note Springer Nature remains neutral with regard to jurisdictional claims in published maps and institutional affiliations.

Open Access This article is licensed under a Creative Commons Attribution 4.0 International License, which permits use, sharing, adaptation, distribution and reproduction in any medium or format, as long as you give appropriate credit to the original author(s) and the source, provide a link to the Creative Commons licence, and indicate if changes were made. The images or other third party material in this article are included in the article's Creative Commons licence, unless indicated otherwise in a credit line to the material. If material is not included in the article's Creative Commons licence and your intended use is not permitted by statutory regulation or exceeds the permitted use, you will need to obtain permission directly from the copyright holder. To view a copy of this licence, visit http://creativecommons.org/licenses/by/4.0/.

(C) The Author(s) 2021 